\title{
Folding Back in Decision Tree Analysis
}

\author{
Rakesh Sarin • Peter Wakker \\ Anderson Graduate School of Management, University of California, 405 Hilgard Avenue, \\ Los Angeles, California 90024-1481 \\ Medical Decision Making Unit, University of Leiden (AZL), Leiden, The Netherlands
}

\begin{abstract}
$\mathrm{T}_{\mathrm{s}}$ his note demonstrates that two minimal requirements of decision tree analysis, the folding back procedure and the interchangeability of consecutive event nodes, imply independence. (Decision Analysis; Dynamic Choice)
\end{abstract}

It is now well-known that under a set of decision tree rationality assumptions expected utility (EU) is the only appropriate model for evaluating alternatives in a decision tree. Early discussions are found in Raiffa (1968) and Burks (1977). Recently, Karni and Schmeidler (1991) formally showed that the reduction of compound lotteries axiom, consequentialism, and dynamic consistency can only be simultaneously satisfied if EU holds. Surprisingly, a much weaker set of assumptions than hitherto presented in the literature is required to justify the use of EU in decision tree analysis. We shall show that a simple rule that is widely used in decision tree constructions, together with the usual folding back procedure, is sufficient to justify the independence principle, the critical condition for EU. Our conditions are more basic than the ones used before in the literature. Further, by invoking a theorem of Gorman (1968), we are able to derive independence from fewer structural assumptions. In particular, our analysis needs the availability of only a few uncertain events, and does not require the existence of extraneously specified probabilities.

LaValle and Wapman (1986) showed that consistency between results obtained by rolling back an extensive tree and its probabilistically equivalent normal tree can only be achieved under independence. LaValle (1992) reaches the same conclusion by requiring invariance and horizon flexibility in alternative representations of decision trees. We obtain independence in a more general context. We require only that the decision maker accept interchangeability of consecutive event nodes and the possibility of folding back as an appropriate method of analysis. The folding-back procedure need only calculate certainty equivalents according to whatever preference model the decision-maker uses, ${ }^{1}$ and does not need given probabilities, or a multiplication rule for those. Our result is therefore obtained with a weaker set of assumptions.

A cardinal rule in the construction of decision trees is that consecutive event nodes can be interchanged or combined. As an example, suppose the profits of an icecream vendor depend on whether the weather is warm $(W)$ or cold $(C)$, and the size of the crowd, big $(B)$ or small $(S)$. Three representations of this problem are given in Figure 1. This figure depicts alternative event trees $^{2}$ that emanate from a given act. The only difference between the representations in Tree 1 and Tree 3 is that consecutive event nodes are interchanged. Regardless of the preference model employed, most people will agree that the three versions in the figure should give equivalent results. We further assume that the decision maker evaluates the decision trees by means of the folding back technique. Thus, at nodes 2 and 3 in Tree 1 , he computes the certainty equivalent of the respective gambles, using any appropriate model of his choice. In

\footnotetext{
${ }^{1}$ We only require the standard assumptions of transitivity, completeness, strict monotonicity (any strict increase of a profit leads to a strict increase in appreciation), and continuity. This permits a large class of nonexpected utility models, such as Choquet expected utility, or, if probabilities are given, rank-dependent utility, weighted utility, lottery-dependent utility, or any Machina functional (see Fishburn, 1988).

${ }^{2}$ An event tree is a decision tree, or a subpart thereof, in which there are only event nodes and no decision nodes.
} 
Figure 1 The Three Event Trees Emanating from the Same Act
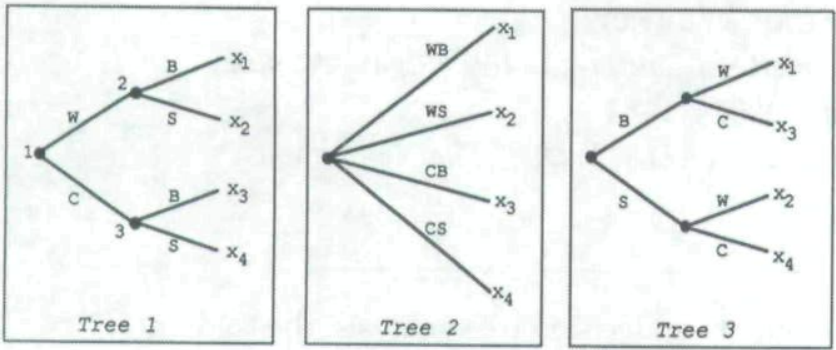

the first stage the gamble over these certainty equivalents is evaluated subsequently. This standard procedure of folding back decision trees assumes that the evaluation at node 2 is independent of the evaluation at node 3 , and vice versa (consequentialism). It further assumes that the certainty equivalents, computed at nodes 2 and 3 , are used in the evaluation at node 1 (dynamic consistency).

The only structural assumption that we use in the result below is that the outcomes $x_{1}, \ldots, x_{4}$ can be any real numbers. ${ }^{3}$ Note that the events in Figure 1 are kept fixed in our analysis, so that the result can be applied to any fixed quadruple of disjoint events $W B, W S, C B$, CS. ${ }^{4}$ Formally, interchangeability means that the three trees in Figure 1 are equivalent in the sense that they induce the same preference relation over quadruples $\left(x_{1}, \ldots, x_{4}\right)$. Thus, only their "normal form" is essential, and the timing and exact sequence of the consecutive events (with no intervening decision nodes) does not matter. Independence in the context of decision making under uncertainty is Savage's (1954) sure-thing principle. It requires that every event is separable, i.e., preferences between acts that coincide outside that event are independent of the particular outcomes of those acts outside the event.

\footnotetext{
${ }^{3}$ This can be greatly generalized. For instance the outcome set can be any nondegenerate interval, and outcomes may be state-dependent in the sense that different intervals are used for different events. Also, the result extends directly to the case in which the outcome set is a convex set of any dimension. This includes the case in which outcomes are probability distributions over a set of prizes, as considered in Hammond (1988), and Anscombe and Aumann (1963).

${ }^{4}$ The assumption that there are only two first-stage events $W, C$, and only two second-stage events $S, B$, was only made for simplicity of presentation. The extension to the case of $m$ first-stage events, and $n$ second-stage events, for any $m \geq 2, n \geq 2$, is immediate.
}

We note that we do not require that probabilities are given for the events. For the special case in which probabilities are given, we do not require that a reduction of compound lotteries assumption holds. The latter assumption was used by LaValle and Wapman (1986), Hammond (1988), and Karni and Schmeidler (1991) in their derivation of independence.

OBSERVATION. Interchangeability of consecutive event nodes, along with the folding back procedure, implies independence.

Proof. Consider the preferences in Tree 2. The possibility to employ folding back in Tree 1, together with the possibility to combine the event nodes of Tree 1 into those of Tree 2, implies separability of the events $\{W B, W S\}$ and $\{C B, C S\}$ in Tree 2 . The possibility to employ folding back in Tree 3, together with the possibility to combine the event nodes of Tree 3 into those of Tree 2, implies separability of the events $\{W B, C B\}$ and $\{W S, C S\}$ in Tree 2. By the theorem of Gorman (1968) all events must then be separable in Tree 2. This is equivalent to independence.

We note that the equivalence between Tree 1 and Tree 2 alone can be preserved by a wide class of nonexpected utility models. To see this, suppose $V_{1}, V_{2}, V_{3}$ are three arbitrary (nonexpected utility) functions from $I R^{2}$ to $I R$, continuous and strictly increasing in both variables. Suppose the decision maker uses $V_{2}$ at node 2 to obtain $V_{2}\left(x_{1}, x_{2}\right)$, and similarly he uses $V_{3}$ at node 3 . Finally, at node 1 the evaluation $V_{1}\left(V_{2}\left(x_{1}, x_{2}\right), V_{3}\left(x_{3}\right.\right.$, $\left.x_{4}\right)$ ) is used. The consistency between Trees 1 and 2 is simply achieved by using the evaluation $V_{1}\left(V_{2}\left(x_{1}, x_{2}\right)\right.$, $\left.V_{3}\left(x_{3}, x_{4}\right)\right)$ in Tree 2 . The only implication of folding back in Tree 1 is that in Tree 2, the events $\{W B, W S\}$ (associated with $\left.\left(x_{1}, x_{2}\right)\right)$ and $\{C B, C S\}$ (associated with $\left.\left(x_{3}, x_{4}\right)\right)$ must be separable. So the reducibility of the two-stage Tree 1 to the one-stage Tree 2 is not very restrictive, and does not imply independence. For the derivation of independence in the above observation, the equivalence with Tree 3 is essential.

Let us comment on the special case of decision making under risk, in which probabilities are given. Segal (1990) argued for abandoning the reduction of compound lotteries assumption in the folding back of decision trees by means of nonexpected utility models. As our analysis has shown, a requirement more basic than the reduction 
of compound lotteries must be abandoned to justify the use of nonexpected utility models in the folding back analysis. What is crucial here is not so much the multiplication operation of probabilities, but, more elementarily, the very possibility of combining and interchanging consecutive event nodes. It may happen that interchangeability is not reasonable in experimental psychology where the order of the presentation of events could indeed influence a subject's perception and evaluation. In the domain of decision analysis, however, this condition seems eminently reasonable. This is because the difference between Trees 1 and 3 is not substantive with respect to the underlying decision problem, and arises as an arbitrary choice of problem representation by the decision analyst.

McClennen (1990) and Machina (1989) suggest a way to achieve equivalence among decision trees such as those in Figure 1. They require that the evaluation at the second-stage node 2 be dependent on the gamble in node 3 , and vice versa. Thus they can keep dynamic consistency and reduction of compound lotteries, but they drop consequentialism. This relaxation of consequentialism, however, precludes the folding back procedure as described above, thus it complicates the analysis of extensive trees.

In a seminal paper, Hammond (1988) provided results similar to the above observation. His term "consequentialism" entails both dynamic consistency as described above and the equivalence of decision trees such as in Figure 1. Also he assumed our consequentialism, named "consistency" in his paper. The main difference is that he assumes a rich model with many structural assumptions, whereas our model is elementary and uses minimal structure. Hammond assumes that all decision trees and all probability distributions are available, and uses these in his derivations. For probabilities he assumes reduction of compound lotteries, and he gives a central role to stochastic independence. Our result has shown that independence can already be derived in the simplest setup, a setup that can be found within small subparts of decision trees. Thus, our paper has reduced the derivation of independence to its bare essence.

Because of the mild structural restrictions that we invoked, our assumptions, while implying independence, have not entirely determined expected utility maximization. This finding shows additionally that the as- sumptions in the above observation are not very restrictive. As demonstrated by Gorman (1968), the conditions in the proposition are necessary and sufficient for additive representability, i.e., the possibility to represent preferences by a function

$$
\left(x_{1}, \ldots, x_{4}\right) \mapsto \sum_{i=1}^{4} \phi_{i}\left(x_{i}\right),
$$

where the $\phi_{i}$ functions are continuous and strictly increasing. This form can be interpreted as state-dependent expected utility, the model advanced by Karni (1985). To obtain expected utility maximization, independence must be slightly strengthened. One such strengthening was proposed in Wakker $(1984,1989)$, and an alternative strengthening was given in Gul (1992). Under the assumption, customary in the literature, that probabilities are available and that the usual symmetry conditions are satisfied, ${ }^{5}$ expected utility maximization follows straightforwardly. In our context, EU follows if the decision maker accepts interchangeability of consecutive event nodes for all possible events, and probabilities are given for all these events. Alternatively, if outcomes are probability distributions over a set of prizes, then a simple monotonicity condition suffices to give EU (see for instance Anscombe and Aumann, 1963).

The point is that the hurdles along the road from independence to EU are relatively small. Nonexpected utility models are designed primarily to permit nonseparability of preferences across mutually exclusive events. We have shown that such nonseparability cannot be permitted in decision tree analysis if one accepts folding back and interchangeability of consecutive event nodes as two reasonable rules.

In summary, the folding back process is a requirement for the standard decision technology that is used to evaluate decision trees. Interchangeability of consecutive event nodes is a requirement for preserving equivalence in alternative representations of the same problem. It is remarkable that these two requirements are sufficient to derive independence. ${ }^{6}$

${ }^{5}\left(\frac{1}{4}, x_{1} ; \frac{1}{4}, x_{2} ; \frac{1}{4}, x_{3} ; \frac{1}{4}, x_{4}\right) \sim\left(\frac{1}{4}, x_{2} ; \frac{1}{4}, x_{1} ; \frac{1}{4}, x_{3} ; \frac{1}{4}, x_{4}\right)$, etc.

${ }^{6}$ The support for this research was provided in part by the Decision, Risk, and Management Science branch of the National Science Foundation. 


\section{References}

Anscombe, F. J. and R. J. Aumann, "A Definition of Subjective Probability," Annals of Mathematical Statistics, 34 (1963), 199-205.

Burks, A. W., Chance, Cause, Reason: An Inquiry into the Nature of Scientific Evidence, The University of Chicago Press, Chicago, IL, 1977.

Fishburn, P. C., Nonlinear Preference and Utility Theory, Johns Hopkins University Press, Baltimore, MD, 1988.

Gorman, W. M., "The Structure of Utility Functions," Review of Economic Studies, 35 (1968), 367-390.

Gul, F., "Savage's Theorem with a Finite Number of States," J. Economic Theory, 57 (1992), 99-110.

Hammond, P. J., "Consequentialist Foundations for Expected Utility," Theory and Decision, 25 (1988), 25-78.

Karni, E., Decision-Making under Uncertainty: The Case of State-Dependent Preferences, Harvard University Press, Cambridge, MA, 1985.

- and D. Schmeidler, "Atemporal Dynamic Consistency and Expected Utility Theory," J. Economic Theory, 54 (1991), 401-408.

LaValle, I. H., "Small Worlds and Sure Things: Consequentialism by the Back Door," in W. Edwards (Ed.), Utility Theories: Measurement and Applications, Kluwer Academic Publishers, Dordrecht, The Netherlands, 1992, 109-136.

- and K. R. Wapman, "Rolling Back Trees Requires the Independence Axiom," Management Sci., 32 (1986), 382-385.

Machina, M. J., "Dynamic Consistency and Non-Expected Utility Models of Choice under Uncertainty," J. Economic Literature, 27 (Dec. 1989b), 1622-1688.

McClennen, E. F., Rationality and Dynamic Choice: Foundational Explorations, Cambridge University Press, Cambridge, England, 1990.

Raiffa, H., Decision Analysis, Addison-Wesley, London, England, 1968. Savage, L. J., The Foundations of Statistics, Wiley, New York, 1954 (Second ed., Dover, New York, 1972.)

Segal, U., "Two-Stage Lotteries without the Reduction Axiom," Econometrica , 58 (1990), 349-377.

Wakker, P. P., "Cardinal Coordinate Independence for Expected Utility," J. Mathematical Psychology, 28 (1984), 110-117.

-, Additive Representations of Preferences: A New Foundation of Decision Analysis, Kluwer Academic Publishers, Dordrecht, The Netherlands, 1989.

Accepted by Robert T. Clemen; received September 9, 1992. This paper has been with the authors 2 months for 1 revision. 
Copyright 1994, by INFORMS, all rights reserved. Copyright of Management Science is the property of INFORMS: Institute for Operations Research and its content may not be copied or emailed to multiple sites or posted to a listserv without the copyright holder's express written permission. However, users may print, download, or email articles for individual use. 